\title{
On the Eulerian enumeration of involutions
}

\author{
Chak-On Chow \\ P.O. Box 91100, Tsimshatsui Post Office, Hong Kong \\ cchow@alum.mit.edu \\ Submitted: Jan 20, 2008; Accepted: Apr 30, 2008; Published: May 12, 2008 \\ Mathematics Subject Classifications: 05A15, 05A20
}

\begin{abstract}
We consider in this work the enumeration of involutions by descent sets, and based on that, by descent numbers. Formulas of the number of involutions with a prescribed descent set, with a prescribed descent number and Frobenius-type formulas are given.
\end{abstract}

\section{Introduction}

This work was motivated by recent work on permutation statistics on involutions $[4,7]$. Dukes [4] proved partially the unimodality of the Eulerian distribution on involutions. The unimodality was later fully established by Guo and Zeng [7].

Several authors have considered enumeration problems closely related to the class of involutions. Désarménien and Foata [3] obtained, amongst other things, a multivariate basic hypergeometric series according to the quadruple (fix, trans, des, maj), where fix $\sigma$, $\operatorname{trans} \sigma$, $\operatorname{des} \sigma$ and maj $\sigma$ denote respectively the number of fixed points, the number of transpositions, the number of descents, and the major index, of $\sigma$, on the class of involutions. Gessel and Reutenauer [6] enumerated permutations according to a prescribed cycle structure and descent set. (Results concerning involutions are immediate by restricting the cycle lengths to at most two.)

Although different kinds of generating functions of involutions exist in the literature, explicit formulas for the number of involutions with prescribed descent sets appear to be missing. It is the purpose of the present work to fill this gap, and in the course of doing so, we obtain the involution analogues of the corresponding results on permutations. The point of departure of this work is the above mentioned work of Gessel and Reutenauer.

The organization of this paper is as follows. In the next section, we collect certain notations and preliminary results to which we shall refer in subsequent sections. In Section 3 , we consider the enumeration of involutions by descent sets. In Section 4 , we demonstrate the decomposition properties of $f_{C}^{\lambda}$, the number of involutions of shape $\lambda$ and of descent composition $C$. In Section 4, we consider the complementary symmetry of the descent 
statistics on involutions. In the final section, we obtain Frobenius-type formulas for the generating functions of involutions by descent numbers.

\section{Notations and preliminaries}

Denote by $\mathbb{Z}$ the set of integers. If $m, n \in \mathbb{Z}$, then $[m, n]=\{m, m+1, \ldots, n\}$, and $[n]:=[1, n]$. The cardinality of a finite set $S$ is denoted by $\# S$.

Denote by $\mathfrak{S}_{n}$ the symmetric group on $n$ letters, which is a Coxeter group of type $A_{n-1}$ of rank $n-1$ generated by $s_{1}, s_{2}, \ldots, s_{n-1}$, where $s_{i}=(i, i+1)$ is the simple transposition exchanging $i$ and $i+1, i=1,2, \ldots, n-1$. We shall respresent any $\sigma \in \mathfrak{S}_{n}$ by the word $\sigma_{1} \sigma_{2} \cdots \sigma_{n}$, where $\sigma_{i}=\sigma(i), i=1,2, \ldots, n$. An integer $i \in[n-1]$ is said to be a descent of $\sigma$ if $\sigma_{i}>\sigma_{i+1}$. Denote by $D(\sigma):=\left\{i: \sigma_{i}>\sigma_{i+1}\right\}$ the descent set, and $d(\sigma):=\# D(\sigma)$ the number of descents (or descent number), of $\sigma$.

A permutation $\sigma=\sigma_{1} \cdots \sigma_{n} \in \mathfrak{S}_{n}$ is alternating (resp., reverse alternating) if $\sigma_{1}<$ $\sigma_{2}>\sigma_{3}<\sigma_{4}>\cdots$ (resp., $\sigma_{1}>\sigma_{2}<\sigma_{3}>\sigma_{4}<\cdots$ ). Equivalently, $\sigma$ is alternating (resp., reverse alternating) if $D(\sigma)=\{2,4, \ldots, 2\lfloor(n-1) / 2\rfloor\}$ (resp., $D(\sigma)=\{1,3, \ldots, 2\lfloor n / 2\rfloor-$ $1\})$. The Coxeter-theoretic length of $\sigma \in \mathfrak{S}_{n}$ is defined as $\ell(\sigma):=\min \left\{r: s_{i_{1}} s_{i_{2}} \cdots s_{i_{r}}=\right.$ $\sigma\}$. Two distinguished elements of $\mathfrak{S}_{n}$ are the identity element $12 \cdots n$, and the Coxeter element $w_{0}:=n(n-1) \cdots 21$. The Coxeter element $w_{0}$ is the longest element in the sense that its Coxeter-theoretic length is the largest among all elements of $\mathfrak{S}_{n}$.

An integer $i \in[n]$ is said to be a fixed point of $\sigma \in \mathfrak{S}_{n}$ if $\sigma_{i}=i$. A permutation $\sigma \in \mathfrak{S}_{n}$ is an involution if $\sigma^{2}$ is the identity permutation $12 \cdots n$. Denote by $I_{n}$ the set of involutions, and $J_{n}$ the set of fixed-point-free involutions, in $\mathfrak{S}_{n}$. It is clear that $J_{2 n-1}=\varnothing$ for $n=1,2, \ldots$.

Let $n$ be a positive integer. A partition of $n$ is a weakly decreasing sequence $\lambda=$ $\left(\lambda_{1}, \lambda_{2}, \ldots, \lambda_{k}\right)$ of positive integers such that $|\lambda|:=\sum_{i=1}^{k} \lambda_{i}=n$. We shall say that $\lambda$ is of weight $n$, has $k$ parts and write $\ell(\lambda)=k$. We also write $\lambda \vdash n$ to denote that $\lambda$ is a partition of $n$. One also writes $\lambda=\left\langle 1^{m_{1}} 2^{m_{2}} \cdots n^{m_{n}}\right\rangle$, where $m_{i}:=\#\left\{j: \lambda_{j}=i\right\}$, the number of parts of $\lambda$ equal to $i$. A composition of $n$ is an ordered sequence of positive integers $C=\left(c_{1}, c_{2}, \ldots, c_{k}\right)$ such that $|C|:=\sum_{i=1}^{k} c_{i}=n$. The positive integers $c_{i}$ are called parts of $C, C$ is said to have $k$ parts, and we write $\ell(C)=k$. We also write $C \models n$ to mean that $C$ is a composition of $n$. A Ferrers diagram of shape $\lambda$ consists of left-justified rows with $\lambda_{1}$ boxes in the first row, $\lambda_{2}$ boxes in the second row, etc. The conjugate partition $\lambda^{\prime}$ of $\lambda$ is the partition whose associated Ferrers diagram is the transpose of that of $\lambda$. A semistandard Young tableau (SSYT) of shape $\lambda$ and of type $\mu=\left(\mu_{1}, \mu_{2}, \ldots, \mu_{n}\right)$ is a Ferrers diagram filled with $\mu_{1} 1$ 's, $\mu_{2} 2$ 's, etc., so that it is weakly increasing along rows from left to right, and strictly increasing along columns from top to bottom. A standard Young tableau (SYT) of shape $\lambda$ is a semistandard Young tableau of shape $\lambda$ and of type $(1,1, \ldots, 1)$, i.e., $1,2, \ldots, n$ each occurs exactly once. Denote by $\operatorname{SYT}(\lambda)$ the set of all standard Young tableaux of shape $\lambda$, and $f^{\lambda}:=\# \operatorname{SYT}(\lambda)$.

Let $T$ be a standard Young tableau. An integer $i$ is said to be a descent of $T$ if $i+1$ appears in a lower row of $T$ than $i$. See, e.g., [12, p. 361]. Denote by $D(T)$ the descent set of $T$. This notion of descent set $D(T)$ for a tableau $T$ is alternatively called the inverse 
line of route, denoted Iligne $T$, by French researchers. See, e.g., [3, §3]. It is clear that transposing a standard Young tableau $T$ of order $n$ yields a standard Young tableau $T^{t}$ of order $n$ with $D\left(T^{t}\right)=[n-1] \backslash D(T)$.

An important combinatorial algorithm is the Robinson-Schensted-Knuth correspondence, a bijective map from $\sigma \in \mathfrak{S}_{n}$ to pairs of standard Young tableaux $(P, Q)$ that have the same shape $\lambda \vdash n$. We shall write $\sigma \stackrel{\text { RSK }}{\longrightarrow}(P, Q)$ to designate a permutation $\sigma$ and its corresponding pair of tableaux $(P, Q)$. It is well known [11] that if $\sigma \stackrel{\mathrm{RSK}}{\longrightarrow}(P, Q)$, then $\sigma^{-1} \stackrel{\mathrm{RSK}}{\longrightarrow}(Q, P)$. It is also well known that, under the Robinson-Schensted-Knuth correspondence, involutions are in bijective correspondence with standard Young tableaux so that results on involutions are readily translated into ones on standard Young tableaux, and vice versa. Note also the remarkable result of Schützenberger [10] that the number of fixed points of $\sigma \in I_{n}$ is equal to the number of columns of $P$ (and/or $Q$ ) of odd length.

Gessel and Reutenauer [6, p. 206] remarked that the Robinson-Schensted algorithm transforms involutions into standard Young tableaux, preserving descents. A more general statement about this descent-preserving property [12, Lemma 7.23.1] is as follows.

Proposition 1. Let $\sigma \in \mathfrak{S}_{n}$ and $\sigma \stackrel{\mathrm{RSK}}{\longrightarrow}(P, Q)$. Then $D(\sigma)=D(Q)$ and $D\left(\sigma^{-1}\right)=D(P)$.

For other aspects of the Robinson-Schensted-Knuth correspondence, see [9, Chapter $3]$ and [12, Chapter 7].

It is well-known that subsets $S=\left\{s_{1}<s_{2}<\cdots<s_{k}\right\}$ of $[n-1]$ are in bijective correspondence with compositions $C=\left(c_{1}, c_{2}, \ldots, c_{k+1}\right)$ of $n$, the correspondence being

$$
\begin{aligned}
s_{i}=c_{1}+c_{2}+\cdots+c_{i}, & i=1,2, \ldots, k, \\
c_{i}=s_{i}-s_{i-1}, & i=1,2, \ldots, k+1,
\end{aligned}
$$

where $s_{0}:=0$ and $s_{k+1}:=n$. We shall write $S(C)$ to denote the subset of $[n-1]$ corresponding to $C$, and $C(S)$ the composition of $n$ corresponding to $S$. It is convenient to transfer the partial order on subsets of $[n-1]$ to compositions of $n$ by defining $C \preccurlyeq$ $D \Longleftrightarrow S(C) \subseteq S(D)$.

Let $X=\left\{x_{1}, x_{2}, \ldots\right\}$ be an infinite alphabet of commuting letters totally ordered by $x_{i}<x_{j} \Longleftrightarrow i<j$. The fundamental quasi-symmetric function $F_{C}$ in the alphabet $X$ indexed by a composition $C$ of $n$ is defined by

$$
F_{C}=\sum_{\substack{1 \leqslant i_{1} \leqslant i_{2} \leqslant \ldots \leqslant i_{n} \\ j \in S(C) \Rightarrow i_{j}<i_{j+1}}} x_{i_{1}} x_{i_{2}} \cdots x_{i_{n}}
$$

We shall suppress the designation of alphabet(s) unless the circumstance demands the contrary. Let QSym $n$ be the $K$-linear span of all $F_{C}$ with $|C|=n$, and QSym $:=\bigoplus_{n \geqslant 0} \operatorname{QSym}_{n}$ its completion, with $\mathrm{QSym}_{0}:=K$ a field of characteristic 0 .

Given a permutation $\sigma=\sigma_{1} \sigma_{2} \cdots \sigma_{n}$ in $\mathfrak{S}_{n}$, its descent composition $C(\sigma)$ is defined as the composition of $n$ corresponding to the descent set $D(\sigma)$ of $\sigma$. 
Let $\mu=\left(\mu_{1}, \mu_{2}, \ldots, \mu_{k}\right)$ be an integer partition. The homogeneous symmetric function $h_{n}$ (in the alphabet $X$ ) is defined by

$$
h_{n}=\sum_{1 \leqslant i_{1} \leqslant i_{2} \leqslant \cdots \leqslant i_{n}} x_{i_{1}} x_{i_{2}} \cdots x_{i_{n}}
$$

and $h_{\mu}=h_{\mu_{1}} h_{\mu_{2}} \cdots h_{\mu_{k}}$; the monomial symmetric function $m_{\mu}$ indexed by $\mu$ is defined by

$$
m_{\mu}=\sum_{1 \leqslant i_{1}<i_{2}<\cdots<i_{k}} x_{i_{1}}^{\alpha_{1}} x_{i_{2}}^{\alpha_{2}} \cdots x_{i_{k}}^{\alpha_{k}}
$$

where the sum is over all distinct permutations $\alpha=\left(\alpha_{1}, \alpha_{2}, \ldots, \alpha_{k}\right)$ of $\left(\mu_{1}, \mu_{2}, \ldots, \mu_{k}\right)$. In the algebra $\Lambda$ of symmetric functions, $h_{\lambda}$ and $m_{\mu}$ form dual bases with respect to the inner product $\langle\cdot, \cdot\rangle$, i.e., $\left\langle h_{\lambda}, m_{\mu}\right\rangle=\delta_{\lambda, \mu}$, and the Schur functions $s_{\lambda}$ form a self-dual basis, i.e., $\left\langle s_{\lambda}, s_{\mu}\right\rangle=\delta_{\lambda, \mu}$. Expressing $s_{\lambda}$ in terms of $m_{\mu}, s_{\lambda}=\sum_{\mu} K_{\lambda, \mu} m_{\mu}$, where $K_{\lambda, \mu}$ is a Kostka number, which counts the number of semistandard Young tableaux (SSYT) of shape $\lambda$ and of type $\mu$.

Let $K$ be a field of characteristic 0 and $x_{1}, x_{2}, \ldots$ be commuting indeterminates. A formal power series $f \in K\left[\left[x_{1}, x_{2}, \ldots\right]\right]$ is called quasi-symmetric if the coefficients of $x_{i_{1}}^{c_{1}} x_{i_{2}}^{c_{2}} \cdots x_{i_{k}}^{c_{k}}$ and $x_{j_{1}}^{c_{1}} x_{j_{2}}^{c_{2}} \cdots x_{j_{k}}^{c_{k}}$ in $f$ are equal, where $i_{1}<i_{2}<\cdots<i_{k}, j_{1}<j_{2}<\cdots<$ $j_{k}$ and $c_{1}, c_{2}, \ldots, c_{k} \geqslant 0$. We shall need the following result of Gessel [5, Theorem 3] concerning coefficient extraction of formal series.

Proposition 2. If a quasi-symmetric formal series $g=\sum_{L} a_{L} F_{L}$ is symmetric, then $a_{L}=\left\langle g, S_{L}\right\rangle$, where $S_{L}=\sum_{K \preccurlyeq L}(-1)^{\ell(L)-\ell(K)} h_{K}$ is a skew Schur function.

\section{Counting involutions by descent sets}

We consider in this section the enumeration of involutions by descent sets. Gessel and Reutenauer [6, eq.(7.1)] obtained the quasi-symmetric generating function for involutions, namely,

$$
\sum_{\pi} F_{C(\pi)} \alpha^{\operatorname{fix}(\pi)} z^{|\pi|}=\prod_{i}\left(1-\alpha z x_{i}\right)^{-1} \prod_{i<j}\left(1-z^{2} x_{i} x_{j}\right)^{-1}
$$

where the sum is over all involutions $\pi, \operatorname{fix}(\pi)$ is the number of fixed points of $\pi$, and $|\pi|$ is the length of the word $\pi$. From the linear Schur formula:

$$
\sum_{\lambda} \alpha^{c(\lambda)} s_{\lambda}=\prod_{i}\left(1-\alpha x_{i}\right)^{-1} \prod_{i<j}\left(1-x_{i} x_{j}\right)^{-1}
$$

where $c(\lambda)$ is the number of columns of $\lambda$ of odd length, the combinatorial definition of Schur functions, and $x_{i} \mapsto z x_{i}$, we have that

$$
\sum_{\lambda} \alpha^{c(\lambda)} s_{\lambda} z^{|\lambda|}=\prod_{i}\left(1-\alpha z x_{i}\right)^{-1} \prod_{i<j}\left(1-z^{2} x_{i} x_{j}\right)^{-1} .
$$


Equating this with (1), followed by extracting the coefficients of $z^{n}$, we have that

$$
\sum_{\pi \in I_{n}} F_{C(\pi)} \alpha^{\mathrm{fix}(\pi)}=\sum_{\lambda \vdash n} \alpha^{c(\lambda)} s_{\lambda}
$$

which is symmetric. Applying Proposition 2 then yields

$$
\begin{aligned}
\sum_{\pi \in I_{n}, C(\pi)=C} \alpha^{\mathrm{fix}(\pi)} & =\left\langle\sum_{\lambda} \alpha^{c(\lambda)} s_{\lambda}, S_{C}\right\rangle \\
& =\left\langle\sum_{\lambda} \alpha^{c(\lambda)} \sum_{\mu} K_{\lambda, \mu} m_{\mu}, \sum_{D \preccurlyeq C}(-1)^{\ell(C)-\ell(D)} h_{D}\right\rangle \\
& =\sum_{\lambda} \sum_{D \preccurlyeq C}(-1)^{\ell(C)-\ell(D)} \alpha^{c(\lambda)} K_{\lambda, D} .
\end{aligned}
$$

In particular, setting $\alpha=1$ yields the following expression for the number of involutions in $\mathfrak{S}_{n}$ with descent composition $C$ :

$$
\#\left\{\pi \in I_{n}: C(\pi)=C\right\}=\sum_{\lambda \vdash n} \sum_{D \preccurlyeq C}(-1)^{\ell(C)-\ell(D)} K_{\lambda, D} .
$$

The sum on the right can be explained combinatorially with the help of $[12$, Exercise 7.90.a], which states that the Kostka number $K_{\lambda / \mu, \alpha}$ is equal to the number of standard Young tableaux $T$ of shape $\lambda / \mu$ satisfying $D(T) \subseteq S(\alpha)$, where $\alpha=\left(\alpha_{1}, \ldots, \alpha_{k}\right)$ is a composition of $n$. Inclusion-exclusion then yields the right hand side, which is equal to the number of standard Young tableaux of order $n$ with descent composition $C$. As the Robinson-Schensted-Knuth correspondence is descent-preserving (hence descent composition-preserving) between involutions and standard Young tableaux, the equality between the left and right sides follows.

Define now the shape $\operatorname{sh}(\sigma)$ of $\sigma \in \mathfrak{S}_{n}$ to be the common shape of the pair $(P, Q)$ of tableaux corresponding to $\sigma$ under the Robinson-Schensted-Knuth correspondence, and $f_{C}^{\lambda}:=\#\left\{\sigma \in I_{n}: \operatorname{sh}(\sigma)=\lambda, C(\sigma)=C\right\}$. We summarize the above in the next theorem.

TheOrem 3. We have

$$
f_{C}^{\lambda}=\sum_{D \preccurlyeq C}(-1)^{\ell(C)-\ell(D)} K_{\lambda, D},
$$

and $\#\left\{\sigma \in I_{n}: C(\sigma)=C\right\}=\sum_{\lambda \vdash n} f_{C}^{\lambda}$, where $K_{\lambda, D}$ is a Kostka number.

\section{Decomposition properties of $f_{C}^{\lambda}$}

In Section 3, we obtained the number $f_{C}^{\lambda}$ of involutions of shape $\lambda$ and descent composition $C$. It is clear that $f_{C}^{\lambda}$ satisfies the following decomposition properties:

$$
\sum_{\lambda \vdash n} f_{C}^{\lambda}=\#\left\{\sigma \in I_{n}: C(\sigma)=C\right\} \quad \text { and } \quad \sum_{C \models n} f_{C}^{\lambda}=f^{\lambda} \text {. }
$$


We shall present in this section one further decomposition property of $f_{C}^{\lambda}$. Toward this end, recall the internal coproduct $\delta:$ QSym $\longrightarrow$ QSym $\otimes$ QSym defined by $\delta f(X) \longrightarrow f(X Y)$, where $f \in$ QSym, and we have identified $f(X Y)=\sum g(X) h(Y)$ with $\sum g(X) \otimes h(Y)$. Here, $X=\left\{x_{1}, x_{2}, \ldots\right\}$ and $Y=\left\{y_{1}, y_{2}, \ldots\right\}$ are infinite totally ordered alphabets of commuting letters, and $X Y=\left\{x_{i} y_{j}\right\}$ is the product alphabet totally ordered by $x_{i} y_{j}<$ $x_{k} y_{l} \Longleftrightarrow(i, j)<(k, l)$ lexicographically. The internal coproduct of fundamental quasisymmetric functions is easy to describe combinatorially [5, Theorem 13], namely, if $\pi \in$ $\mathfrak{S}_{n}$, then

$$
\delta F_{C(\pi)}=F_{C(\pi)}(X Y)=\sum_{\tau \sigma=\pi} F_{C(\sigma)}(X) \otimes F_{C(\tau)}(Y)
$$

where the sum on the right is over all pairs $(\sigma, \tau) \in \mathfrak{S}_{n} \times \mathfrak{S}_{n}$ such that $\tau \sigma=\pi$.

A fundamental identity in the representation theory of symmetric groups is the following [9, Theorem 2.6.5 (3)], [12, (7.43)]:

$$
\sum_{\lambda \vdash n}\left(f^{\lambda}\right)^{2}=n !
$$

of which a bijective proof is provided by the Robinson-Schensted-Knuth correspondence, and a representation-theoretic proof follows from a dimensional consideration of the fact that $\mathbb{C}\left[\mathfrak{S}_{n}\right]=\bigoplus_{\lambda \vdash n} f^{\lambda} S^{\lambda}$, where $\mathbb{C}\left[\mathfrak{S}_{n}\right]$ carries the regular representation of $\mathfrak{S}_{n}$ and $S^{\lambda}$ is the Specht module indexed by $\lambda$ with $\operatorname{dim} S^{\lambda}=f^{\lambda}$.

Substituting $\sum_{C \models n} f_{C}^{\lambda}=f^{\lambda}$ into (2) yields the following decomposition property of $f_{C}^{\lambda}$ :

$$
\sum_{C, D \models n} \sum_{\lambda \vdash n} f_{C}^{\lambda} f_{D}^{\lambda}=n !
$$

The next proposition gives a combinatorial interpretation to $\sum_{\lambda \vdash n} f_{C}^{\lambda} f_{D}^{\lambda}$.

THEOREM 4. We have

$$
\#\left\{\sigma \in \mathfrak{S}_{n}: C(\sigma)=C, C\left(\sigma^{-1}\right)=D\right\}=\sum_{\lambda \vdash n} f_{C}^{\lambda} f_{D}^{\lambda},
$$

where $C, D \models n$.

Proof. Let $\pi=12 \cdots n$, the identity element of $\mathfrak{S}_{n}$, so that $\tau \sigma=\pi \Longleftrightarrow \tau=\sigma^{-1}$. It follows that

$$
\begin{aligned}
\delta F_{C(\pi)} & =\sum_{\sigma \in \mathfrak{S}_{n}} F_{C(\sigma)}(X) \otimes F_{C\left(\sigma^{-1}\right)}(Y) \\
& =\sum_{\lambda \vdash n} \sum_{P, Q \in \mathrm{SYT}(\lambda)} F_{C(Q)}(X) \otimes F_{C(P)}(Y) \\
& =\sum_{\lambda \vdash n} \sum_{C, D \models n} f_{C}^{\lambda} f_{D}^{\lambda} F_{C}(X) \otimes F_{D}(Y),
\end{aligned}
$$

where $\sigma \stackrel{\text { RSK }}{\longrightarrow}(P, Q)$. The proposition now follows upon equating coefficients of $F_{C}(X) \otimes$ $F_{D}(Y)$. 
The number $\#\left\{\sigma \in \mathfrak{S}_{n}: C(\sigma)=C, C\left(\sigma^{-1}\right)=D\right\}$ can also be expressed as the inner product $\left\langle S_{C}, S_{D}\right\rangle$ of two skew Schur functions $S_{C}$ and $S_{D}$. See, e.g., [12, Corollary 7.23.8][5, Theorem 5] for details.

\section{Complementary symmetry}

We consider in this section complementary symmetries of the descent statistic for involutions with and without fixed points.

In case of permutations, the following complementary symmetry holds:

$$
\#\left\{\sigma \in \mathfrak{S}_{n}: D(\sigma)=S\right\}=\#\left\{\sigma \in \mathfrak{S}_{n}: D(\sigma)=[n-1] \backslash S\right\},
$$

where $S \subseteq[n-1]$. A bijective proof of (3) is readily given by either of the two maps $\sigma \longrightarrow \sigma w_{0}$ and $\sigma \longrightarrow w_{0} \sigma$ which sends bijectively a permutation $\sigma \in \mathfrak{S}_{n}$ to a permutation $\sigma^{\prime} \in \mathfrak{S}_{n}$ with descent set $[n-1] \backslash D(\sigma)$. These two maps, unfortunately, do not send involutions to involutions since, for instance, $\sigma w_{0}$ is an involution if and only if $w_{0} \sigma=$ $\left(\sigma w_{0}\right)^{-1}=\sigma w_{0}$, which need not hold for all involution $\sigma$ in $\mathfrak{S}_{n}$.

Proposition 5. The following complementary symmetry holds for involutions:

$$
\#\left\{\sigma \in I_{n}: D(\sigma)=S\right\}=\#\left\{\sigma \in I_{n}: D(\sigma)=[n-1] \backslash S\right\},
$$

where $S \subseteq[n-1]$.

Proof. The map $\sigma \stackrel{\text { RSK }}{\longrightarrow} Q \longrightarrow Q^{t} \stackrel{\mathrm{RSK}^{-1}}{\longrightarrow} \sigma^{\prime}$ sending $\sigma \in I_{n}$ with $D(\sigma)=S$ to $\sigma^{\prime} \in I_{n}$ with $D\left(\sigma^{\prime}\right)=[n-1] \backslash S$ is bijective.

The same proof can be found in $[4,13]$. It is included here to make our discussion selfcontained. Eq. (4) is a refinement of the symmetry result $I_{n, s-1}=I_{n, n-s}$ for $s=1,2, \ldots, n$. For an alternate proof of this symmetry, see [3, Section 6]. For a (quasi-)symmetric generating function proof of (4), see [6, Theorem 4.2].

COROLlaRY 6. Alternating involutions and reverse alternating involutions are equinumerous in $I_{n}$, where $n \geqslant 1$.

An alternative bijective proof of this result for odd $n$ may also be given. Before embarking on the proof, we need some further notations and results. Given a standard Young tableau $Q$ of order $n$, denote by $\operatorname{evac}(Q)$ the evacuation tableau for $Q$. We only need the properties of evacuation here. For the actual definition, see [9, §3.9]. The map $Q \longrightarrow \operatorname{evac}(Q)$ is called the Schützenberger involution [12, Proposition A1.2.9].

If $\sigma=\sigma_{1} \sigma_{2} \cdots \sigma_{n} \in \mathfrak{S}_{n}$, then let $\sigma^{\#}:=\left(n+1-\sigma_{n}\right) \cdots\left(n+1-\sigma_{2}\right)\left(n+1-\sigma_{1}\right)=w_{0} \sigma w_{0}$. It is easy to see that if $\sigma$ is an involution, then so is $\sigma^{\#}$. Also, if $\sigma \stackrel{\text { RSK }}{\longrightarrow}(P, Q)$, then $\sigma^{\#} \stackrel{\operatorname{RSK}}{\longrightarrow}(\operatorname{evac}(P), \operatorname{evac}(Q))$.

Proposition 7. We have $D(\operatorname{evac}(Q))=\{n-i: i \in D(Q)\}$. 
Proof. Let $\sigma \stackrel{\operatorname{RSK}}{\longrightarrow}(P, Q)$. For any $i, i \in D(\sigma) \Longleftrightarrow \sigma_{i}>\sigma_{i+1} \Longleftrightarrow n+1-\sigma_{i+1}>$ $n+1-\sigma_{i} \Longleftrightarrow \sigma_{n-i}^{\#}>\sigma_{n-i+1}^{\#} \Longleftrightarrow n-i \in D\left(\sigma^{\#}\right)$. It follows that $\{n-i: i \in D(Q)\}=$ $\{n-i: i \in D(\sigma)\}=D\left(\sigma^{\#}\right)=D(\operatorname{evac}(Q))$, where the first and the last equivalences follow from Proposition 1.

For example, we have

$$
Q=\begin{array}{rrrr}
1 & 3 & 4 & 7 \\
2 & 5 & & \\
6 & & &
\end{array} \quad D(Q)=\{1,4,5\} ; \quad \operatorname{evac}(Q)=\begin{array}{rrrr}
1 & 2 & 5 & 6 \\
3 & 7 & & \\
4 & & &
\end{array}
$$

It is convenient to express the preceding proposition simply as: $D(\operatorname{evac}(Q))=n-D(Q)$ if $D(Q) \neq \varnothing$, and $\varnothing$ otherwise. By conjugating with the Robinson-Schensted-Knuth correspondence, i.e., $\sigma \stackrel{\text { RSK }}{\longrightarrow} Q \longrightarrow \operatorname{evac}(Q) \stackrel{\mathrm{RSK}^{-1}}{\longrightarrow} \sigma^{\#}$, we obtain an involution, which we again call the Schützenberger involution, on $I_{n}$.

COROLlary 8. The Schützenberger involution is a bijection on $I_{n}$ sending alternating involutions onto alternating (resp., reverse alternating) involutions if $n$ is even (resp., $o d d)$.

The Schützenberger involution is a bijection distinct from the one employed in the proof of Proposition 5. For instance, for $\sigma=2143657 \in I_{7}$, we have $D(\sigma)=\{1,3,5\}$ and

$$
\sigma=2143657 \stackrel{\text { RSK }}{\longrightarrow} Q=\begin{array}{llll}
1 & 3 & 5 & 7 \\
2 & 4 & 6
\end{array} \longrightarrow Q^{t}=\begin{array}{ll}
1 & 2 \\
3 & 4 \\
5 & 6 \\
7
\end{array} \stackrel{\text { RSK }^{-1}}{\longrightarrow} 5736142 \neq 1325476=\sigma^{\#}
$$

where $D(5736142)=D(1325476)=\{2,4,6\}$.

The above complementary symmetry need not hold for involutions without fixed point, the reason being that any $\sigma \in J_{2 n}$ is of $\operatorname{shape} \operatorname{sh}(\sigma)$ a partition of $2 n$ whose conjugate partition consists of all parts even; $\operatorname{sh}(\sigma)$ itself need not have all parts even. It is clear that the bijection in the proof of Proposition 5 is shape-conjugating, and in particular establishes the next corollary.

COROLlaRY 9. The following shape-preserving complementary symmetries hold:

$$
\#\left\{\sigma \in I_{n}: \operatorname{sh}(\sigma)=\lambda, D(\sigma)=S\right\}=\#\left\{\sigma \in I_{n}: \operatorname{sh}(\sigma)=\lambda, D(\sigma)=[n-1] \backslash S\right\}
$$

for self-conjugating $\lambda \vdash n$ and $S \subseteq[n-1]$, and

$$
\begin{aligned}
& \#\left\{\sigma \in J_{2 n}: \operatorname{sh}(\sigma)=\mu \in \mathcal{E}(2 n), D(\sigma)=R\right\} \\
& \quad=\#\left\{\sigma \in J_{2 n}: \operatorname{sh}(\sigma)=\mu \in \mathcal{E}(2 n), D(\sigma)=[2 n-1] \backslash R\right\}
\end{aligned}
$$

for self-conjugating $\mu \vdash 2 n$ and $R \subseteq[2 n-1]$, where $\mathcal{E}(n)$ denotes the set of all partitions of $n$ all of whose parts are even. 
Descent classes of $J_{2 n}$ are in general not symmetric with respect to the usual complementation. However, Strehl [13] had shown that to each $\sigma \in J_{2 n}$ there corresponds bijectively a $\sigma^{\prime} \in J_{2 n}$ such that $\# D\left(\sigma^{\prime}\right)=2 n-\# D(\sigma)$. An immediate consequence of this "complementary" symmetry is the symmetry result $J_{2 n, s}=J_{2 n, 2 n-s}$, where $s=$ $1,2, \ldots, n-1$ (see $[3,6,13])$.

\section{Frobenius-type formulas}

We derive Frobenius-type formulas for involutions in this section. Let

$$
I_{n}(t):=\sum_{\sigma \in I_{n}} t^{d(\sigma)}=\sum_{s=1}^{n} I_{n, s-1} t^{s-1}, \quad \text { and } \quad J_{n}(t):=\sum_{\sigma \in J_{n}} t^{d(\sigma)}=\sum_{s=1}^{n-1} J_{n, s} t^{s},
$$

where $I_{n, s-1}:=\#\left\{\sigma \in I_{n}: d(\sigma)=s-1\right\}$ and $J_{n, s}:=\#\left\{\sigma \in J_{n}: d(\sigma)=s\right\}$. Values of $I_{n}(t)$ and $J_{n}(t)$ for $n \leqslant 6$ can be found in [7, Table 1].

Based on the generating functions of $I_{n}(t)$ and $J_{n}(t)$ :

$$
\begin{gathered}
\sum_{n \geqslant 0} I_{n}(t) \frac{u^{n}}{(1-t)^{n+1}}=\sum_{r \geqslant 0} \frac{t^{r}}{(1-u)^{r+1}\left(1-u^{2}\right)^{r(r+1) / 2}}, \\
\sum_{n \geqslant 0} J_{n}(t) \frac{u^{n}}{(1-t)^{n+1}}=\sum_{r \geqslant 0} \frac{t^{r}}{\left(1-u^{2}\right)^{r(r+1) / 2}},
\end{gathered}
$$

obtained by Désarménien and Foata [3], Guo and Zeng [7] derived linear recurrences for $I_{n}(t)$ and $J_{2 n}(t)$, namely,

$$
\begin{aligned}
n I_{n}(t)= & t(1-t) I_{n-1}^{\prime}(t)+(1+(n-1) t) I_{n-1}(t)+t^{2}(1-t)^{2} I_{n-2}^{\prime \prime}(t) \\
& +t(1-t)(3+(2 n-5) t) I_{n-2}^{\prime}(t)+(n-1)\left(1+t+(n-2) t^{2}\right) I_{n-2}(t),
\end{aligned}
$$

and

$$
\begin{aligned}
2 n J_{2 n}(t)= & t^{2}(1-t)^{2} J_{2 n-2}^{\prime \prime}(t)+\left[2(2-2 n) t^{3}+2(2 n-3) t^{2}+2 t\right] J_{2 n-2}^{\prime}(t) \\
& +\left[2 n(2 n-2) t^{2}+2 t+(2 n-2)\right] J_{2 n-2}(t) .
\end{aligned}
$$

By exploiting Theorem 3, we obtain alternate formulas for $I_{n}(t)$ and $J_{2 n}(t)$.

THEOREM 10. We have

(i) $I_{n, s-1}=\sum_{k=1}^{s}(-1)^{s-k}\left(\begin{array}{c}n-k \\ s-k\end{array}\right) \sum_{\lambda \vdash n, \ell(D)=k} K_{\lambda, D}$;

(ii) $I_{n}(t)=\sum_{k=1}^{n} \sum_{\lambda \vdash n, \ell(C)=k} K_{\lambda, C} t^{k-1}(1-t)^{n-k}$.

Proof. Since \# $\left.\# \sigma \in I_{n}: C(\sigma)=C\right\}=\sum_{\lambda \vdash n, D \preccurlyeq C}(-1)^{\ell(C)-\ell(D)} K_{\lambda, D}$, it follows that

$$
I_{n, s-1}=\sum_{\ell(C)=s} \sum_{\lambda \vdash n, D \preccurlyeq C}(-1)^{\ell(C)-\ell(D)} K_{\lambda, D}=\sum_{k=1}^{s}(-1)^{s-k} \sum_{\lambda \vdash n, \ell(D)=k} \sum_{D \preccurlyeq C, \ell(C)=s} K_{\lambda, D} .
$$


Since there are $\left(\begin{array}{c}n-k \\ s-k\end{array}\right)(s-k)$-subsets $T$ of $[n-1] \backslash S(D)$ for which $S(C)=S(D) \cup T$ with $\ell(C)=s$ and $C \succcurlyeq D$, the right hand side of (9) can be written as

$$
I_{n, s-1}=\sum_{k=1}^{s}(-1)^{s-k}\left(\begin{array}{l}
n-k \\
s-k
\end{array}\right) \sum_{\lambda \vdash n, \ell(D)=k} K_{\lambda, D},
$$

which is (i). By replacing (i) in $I_{n}(t)=\sum_{s=1}^{n} I_{n, s-1} t^{s-1}$ and rearranging, we get (ii).

Theorem 10(ii) is a Frobenius-type formula for $I_{n}(t)$, the one for the classical Eulerian polynomial [2, Theorem E, p. 244] being:

$$
A_{n}(t)=\sum_{k=1}^{n} k ! S(n, k) t^{k}(1-t)^{n-k},
$$

where $S(n, k)$ is a Stirling number of the second kind.

It is clear that the numbers $g_{n, k}:=\sum_{\lambda \vdash n, \ell(C)=k} K_{\lambda, C}$ are non-negative, and admit the combinatorial interpretation:

$$
g_{n, k}=\#\left(\coprod_{\ell(C)=k}\left\{\sigma \in I_{n}: C(\sigma) \preccurlyeq C\right\}\right),
$$

where $\coprod$ denotes disjoint union. It is of interest to compute $g_{n, k}$ by some other means rather than by the combinatorial definition of $K_{\lambda, C}$. Let $G_{n}(t):=\sum_{k=1}^{n} g_{n, k} t^{k-1}$. Theorem 10(ii) can be written as

$$
I_{n}(t)=(1-t)^{n-1} G_{n}\left(\frac{t}{1-t}\right)
$$

Proposition 11. We have

$$
\begin{aligned}
n G_{n}(y)= & (1+2 y) G_{n-1}(y)+y(1+y) G_{n-1}^{\prime}(y)+[6 y(1+y)+n-1] G_{n-2}(y) \\
& +3 y(1+y)(1+2 y) G_{n-2}^{\prime}(y)+y^{2}(1+y)^{2} G_{n-2}^{\prime \prime}(y) \\
n g_{n, k}= & k\left(g_{n-1, k}+g_{n-1, k-1}\right)+\left(k^{2}+n-2\right) g_{n-2, k} \\
& +k(2 k-1) g_{n-2, k-1}+k(k-1) g_{n-2, k-2} .
\end{aligned}
$$

Proof. From (10) we have $I_{n}^{\prime}(t)=(1-n)(1-t)^{n-2} G_{n}(t /(1-t))+(1-t)^{n-3} G_{n}^{\prime}(t /(1-t))$, etc. Substituting $I_{n}(t), I_{n-1}(t), I_{n-2}(t)$ and their derivatives in $(7)$, followed by the replacement $t \longrightarrow y /(1+y)$, and after some algebra, the first assertion follows. By extracting the coefficient of $y^{k-1}$, the second assertion follows.

Recall that $\mathcal{E}(n)$ denotes the set of all partitions of $n$ whose parts are all even. If $\operatorname{sh}(\sigma)=\lambda$, then $\sigma$ is fixed-point-free $\Longleftrightarrow \lambda^{\prime} \in \mathcal{E}(n)$; we readily obtain the analogue of Theorem 10 for $J_{2 n}(t)$, whose proof is omitted. 
THEOREM 12. We have

(i) $J_{2 n, s}=\sum_{k=1}^{s}(-1)^{s-k}\left(\begin{array}{c}2 n-1-k \\ s-k\end{array}\right) \sum_{\lambda \vdash 2 n, \lambda^{\prime} \in \mathcal{E}(2 n), \ell(D)=k+1} K_{\lambda, D}$;

(ii) $J_{2 n}(t)=\sum_{k=1}^{2 n-1} \sum_{\lambda \vdash 2 n, \lambda^{\prime} \in \mathcal{E}(2 n), \ell(C)=k+1} K_{\lambda, C} t^{k}(1-t)^{2 n-1-k}$.

Let $h_{2 n, k}:=\sum_{\lambda \vdash 2 n, \lambda^{\prime} \in \mathcal{E}(2 n), \ell(C)=k+1} K_{\lambda, C}$, and $H_{2 n}(t):=\sum_{k=1}^{2 n-1} h_{2 n, k} t^{k}$ so that

$$
J_{2 n}(t)=(1-t)^{2 n-1} H_{2 n}\left(\frac{t}{1-t}\right) \text {. }
$$

It is immediate that $h_{2 n, k}$ can be combinatorially interpreted as

$$
h_{2 n, k}=\#\left(\coprod_{\ell(C)=k+1}\left\{\sigma \in J_{2 n}: C(\sigma) \preccurlyeq C\right\}\right) \text {. }
$$

By the same procedure as for $I_{n}(t)$, we have the next proposition, whose proof is omitted.

Proposition 13. We have

$$
\begin{aligned}
2 n H_{2 n}(y)= & 2(n+(y+1)(3 y-1)) H_{2 n-2}(y)+2 y(1+y)(1+3 y) H_{2 n-2}^{\prime}(y) \\
& +y^{2}(1+y)^{2} H_{2 n-2}^{\prime \prime}(y) ; \\
2 n h_{2 n, k}= & {[2(n-1)+k(k+1)] h_{2 n-2, k}+2 k(k+1) h_{2 n-2, k-1}+k(k+1) h_{2 n-2, k-2} . }
\end{aligned}
$$

Proposition 14. We have

$$
\begin{aligned}
& \sum_{n \geqslant 0} G_{n}(x) u^{n}=\frac{1}{x^{2}} \sum_{r \geqslant 0}\left(\frac{x}{1+x}\right)^{r+2} \frac{1}{(1-u)^{r+1}\left(1-u^{2}\right)^{r(r+1) / 2}} \\
& \sum_{n \geqslant 0} H_{n}(x) u^{n}=\frac{1}{x^{2}} \sum_{r \geqslant 0}\left(\frac{x}{1+x}\right)^{r+2} \frac{1}{\left(1-u^{2}\right)^{r(r+1) / 2}},
\end{aligned}
$$

where $G_{0}(x)=H_{0}(x):=1 /(1+x)$.

Proof. Let $x=t /(1-t)$. Then $t=x /(1+x)$ so that (10) yields

$$
G_{n}(x)=(1+x)^{n-1} I_{n}\left(\frac{x}{1+x}\right),
$$

where $n \geqslant 1$. Since $I_{0}(t)=1$, we define $G_{0}(x)=1 /(1+x)$. Multiplying now (11) by $u^{n}$, followed by summing over $n \geqslant 0$, we have

$$
\begin{aligned}
\sum_{n \geqslant 0} G_{n}(x) u^{n} & =\frac{1}{(1+x)^{2}} \sum_{n \geqslant 0} \frac{I_{n}\left(\frac{x}{1+x}\right) u^{n}}{\left(1-\frac{x}{1+x}\right)^{n+1}} \\
& =\frac{1}{(1+x)^{2}} \sum_{r \geqslant 0}\left(\frac{x}{1+x}\right)^{r} \frac{1}{(1-u)^{r+1}\left(1-u^{2}\right)^{r(r+1) / 2}} \\
& =\frac{1}{x^{2}} \sum_{r \geqslant 0}\left(\frac{x}{1+x}\right)^{r+2} \frac{1}{(1-u)^{r+1}\left(1-u^{2}\right)^{r(r+1) / 2}},
\end{aligned}
$$


where the second equality follows from (5). The proof of the second assertion, being similar, is omitted.

Note that $G_{n}(t)$ need not be real-rooted for all $n$. Otherwise, (11) would imply the real-rootedness of $I_{n}(t)$ so that Newton's inequality [8, p. 104] would imply the logconcavity of $I_{n}(t)$ (resp., $J_{2 n}(t)$ ), which is false [1]. Similar arguments yield that $H_{2 n}(t)$ need not be real-rooted.

The first few Frobenius expansions of $I_{n}(t)$ and $J_{2 n}(t)$ are listed as follows:

$$
\begin{aligned}
I_{1}(t)= & 1 \\
I_{2}(t)= & (1-t)+2 t \\
I_{3}(t)= & (1-t)^{2}+4 t(1-t)+4 t^{2} \\
I_{4}(t)= & (1-t)^{3}+7 t(1-t)^{2}+15 t^{2}(1-t)+10 t^{3}, \\
J_{2}(t)= & t \\
J_{4}(t)= & t(1-t)^{2}+3 t^{2}(1-t)+3 t^{3}, \\
J_{6}(t)= & t(1-t)^{4}+7 t^{2}(1-t)^{3}+22 t^{3}(1-t)^{2}+30 t^{4}(1-t)+15 t^{5}, \\
J_{8}(t)= & t(1-t)^{6}+12 t^{2}(1-t)^{5}+72 t^{3}(1-t)^{4}+225 t^{4}(1-t)^{3}+375 t^{5}(1-t)^{2} \\
& +315 t^{6}(1-t)+105 t^{7} .
\end{aligned}
$$

Acknowledgements. The author thanks I. Gessel and V. Strehl for providing an expanded version of [13].

\section{References}

[1] M. Barnabei, F. Bonetti and M. Silimbani, A combinatorial setting for involutions and semistandard Young tableaux, arXiv:0704.3509.

[2] L. Comtet, Advanced Combinatorics, D. Reidel, Dordrecht, 1974.

[3] J. Désarménien and D. Foata, Fonctions symétriques et séries hypergéométriques basiques multivariées, Bull. Soc. Math. France 113 (1985), no. 1, 3-22.

[4] W. M. B. Dukes, Permutation statistics on involutions, European J. Combin. 28 (2007), 186-198.

[5] I. M. Gessel, Multipartite $P$-partitions and inner products of skew Schur functions, Contemp. Math. 34 (1984), 289-301.

[6] I. M. Gessel and C. Reutenauer, Counting permutations with given cycle structure and descent set, J. Combin. Theory Ser. A 64 (1993), 189-215.

[7] V. J. W. Guo and J. Zeng, The Eulerian distribution on involutions is indeed unimodal, J. Combin. Theory Ser. A 113 (2006), 1061-1071.

[8] G. H. Hardy, J. E. Littlewood and G. Pólya, Inequalities, Cambridge University Press, Cambridge, 1952. 
[9] B. E. Sagan, The Symmetric Group: Representations, Combinatorial Algorithms, and Symmetric Functions, second edition, Springer, New York, 2001.

[10] M.-P. Schützenberger, La correspondance de Robinson, in: "Combinatoire et représentation du groupe symétrique," Lecture Notes in Mathematics, vol. 579, Springer-Verlag, New York/Berlin, 59-113, 1977.

[11] M.-P. Schützenberger, Quelques remarques sur une construction de Schensted, Math. Scand. 12 (1963), 117-128.

[12] R. P. Stanley, Enumerative Combinatorics, vol. 2, Cambridge University Press, New York, 1999.

[13] V. Strehl, Symmetric Eulerian distributions for involutions, Sém. Lothar. Combin. 1, Strasbourg 1980, Publications de l'I.R.M.A. 140/S-02, Strasbourg, 1981. 